\title{
Bead-sort algorithm for load shuffling in miniload AS/RS with an open-rack structure
}

\begin{abstract}
Automated storage and retrieval systems (AS/RSs) are a combination of equipment and control systems which handle, store and retrieve materials with great speed and accuracy, under a defined degree of automation. AS/RSs are warehousing systems that are widely used in distribution and production environments to manage products with cost-effective utilization of time, space and equipment. This paper presents an open-rack structure with unidirectional-upward mobile loads within the rack, for miniload AS/RS. Bead-sort algorithm and a cellular automaton (CA) are used for defining and simulating of load shuffling in this AS/RS, respectively. Heuristic models are developed for load shuffling and travel time of the storage platform. A cellular automaton is a suitable choice for simulating natural physical systems because it is massively parallel, self-organizing and is driven by a set of simple, local rules. The travel time and performance of proposed AS/RS are analyzed using Monte Carlo simulation and are compared with a conventional one. The results show that the open-rack AS/RS represents a higher performance and the proposed models are reliable for the design and analysis of this kind of AS/RS.
\end{abstract}

Keyword: Automated storage and retrieval systems (AS/RS); Open-rack structure; Load shuffling; Bead-sort algorithm; Cellular automaton (CA); Monte Carlo simulation 